\title{
Rapid diagnosis of micrometastasis of gastric cancer using reverse transcription loop-mediated isothermal amplification
}

\author{
YORIHIKO MUTO $^{1}$, HISAHIRO MATUBARA ${ }^{1}$, TOHRU TANIZAWA ${ }^{2}$, YOSHIHIRO NABEYA ${ }^{1}$, \\ HIROSHI KAWAHIRA ${ }^{1}$, TAKASHI AKAI ${ }^{1}$, ISAMU HOSHINO ${ }^{1}$ and HIDEKI HAYASHI ${ }^{1,3}$ \\ Departments of ${ }^{1}$ Frontier Surgery, and ${ }^{2}$ Basic Pathology, Graduate School of Medicine, Chiba \\ University, 1-8-1 Inohana, Chuo-ku, Chiba 260-8670; ${ }^{3}$ Research Center for Frontier Medical \\ Engineering, Chiba University, 1-33 Yayoi-cho, Inage-ku, Chiba 263-8522, Japan
}

Received May 11, 2011; Accepted June 14, 2011

DOI: $10.3892 /$ or.2011.1389

\begin{abstract}
Methods to detect metastases in biopsy specimens with certain rapidity and accuracy are essential to performing tailor-made surgeries for solid malignancies. Reverse transcription loop-mediated isothermal amplification (RT-LAMP) reaction is a novel technique for detecting mRNA expression of target sequences with high sensitivity and rapidity, even from crude samples without RNA purification. Applicability to detect lymph node ( $\mathrm{LN}$ ) micrometastasis of gastric cancer was tested. Total of $26 \mathrm{LNs}$ were retrieved from 10 patients with primary gastric cancer. Each LN was serially sectioned, and every set of three serial sections were tested for routine histopathological (H\&E) and immunohistochemical examination with anti-cytokeratin antibodies (IHC), and RT-LAMP analysis targeted cytokeratin 19 mRNA. Results from H\&E/IHC and RT-LAMP analysis were compared in each set of sections. All the sections of those containing metastatic lesions equivalent to a volume of overt metastasis (maximum diameter $>2 \mathrm{~mm}$ ), $90 \%$ of those containing micrometastasis (between 2 and $0.2 \mathrm{~mm}$ ) and $83 \%$ of those containing isolated tumor cells $(<0.2 \mathrm{~mm})$ were detectable using this procedure. Total analysis from lysates of clinical specimens required $<75 \mathrm{~min}$. This new technique is suggested to be an alternative to rapid diagnosis of micrometastasis based on conventional histopathological analysis.
\end{abstract}

\section{Introduction}

The concept of sentinel lymph node (SLN), presumably the first site of metastasizing tumor cells via the lymphatic system, emerged in the middle of the last century, and so-called tailor-

Correspondence to: Dr Hideki Hayashi, Research Center for Frontier Medical Engineering, Chiba University, 1-33 Yayoi-cho, Inage-ku, Chiba 263-8522, Japan

E-mail: hhayashi@faculty.chiba-u.jp

Key words: cytokeratin 19, isolated tumor cells, lymph nodes made surgery based on this concept has been conducted in the field of breast cancer and cutaneous malignant melanoma (1-3). Validity of the same concept has been tested in the field of gastrointestinal cancers as well, and favorable results are reported on the basis of conventional histopathological analysis and/or immunohistochemical (IHC) analysis of specimens (4-6). Some of the major gastrointestinal surgeries are well known to cause unavoidable systemic disorders after surgery, and as a result the applicability of less invasive treatments according to individual tumor extent is now under investigation. The SLN concept could provide valuable information that might assist in making such tailor-made surgery a reality in the field of gastrointestinal cancers as well. In the meantime, some authors have introduced assay techniques based on reverse transcriptase-polymerase chain reaction (RT-PCR) to detect metastasis in regional lymph nodes (LNs) of gastric cancers (7-12), and they showed a higher incidence of revealing minor tumor deposits in LNs compared with the technique of routine hematoxylin and eosin (H\&E) or IHC examination. The SLN concept has also been shown to be applicable to gastric cancer even when metastasis is analyzed by RT-PCR, suggesting that tailor-made gastric cancer surgeries should be performed with micrometastasis-detecting techniques of high sensitivity such as RT-PCR. However, the technique requires complex procedures and at least several hours to obtain a final result, and thus such surgeries have not yet been widely accepted, because local or LN recurrence of gastrointestinal tumors results in grim survival for patients. As a consequence, a metastasisdetecting technique with a similar sensitivity and performance as RT-PCR is eagerly awaited.

Loop-mediated isothermal amplification (LAMP) is a novel DNA amplification technique first reported by Notomi et al (13). It employs a strand displacement DNA polymerase and a set of specially designed primers recognizing different regions in the targeted DNA sequence, and this technique amplifies a target DNA with high specificity, efficiency and rapidity under isothermal conditions. This technique is also applicable to RNA upon use of reverse transcriptase together with DNA polymerase (reverse transcriptase-LAMP: RT-LAMP) $(14,15)$. Amplification of a targeted gene is detectable in real-time fashion by an increase of turbidity of the solution derived from a side product of the reactions. Thus, the reaction could be 
Table I. Clinicopathological features of patients enrolled in this study.

\begin{tabular}{|c|c|c|c|c|c|c|c|c|}
\hline Case & Gender & Age & $\mathrm{pT}$ & $\mathrm{pN}$ & Histological type & No. of LN tested & No. of LN HE/IHC(+) & No. of LN RT-LAMP(+) \\
\hline 1 & Male & 63 & 3 & 2 & poor & 4 & 4 & 4 \\
\hline 2 & Male & 62 & 2 & 1 & tub & 6 & 4 & 6 \\
\hline 3 & Female & 67 & 2 & 1 & muc & 1 & 1 & 1 \\
\hline 4 & Male & 63 & 3 & 2 & poor & 4 & 3 & 3 \\
\hline 5 & Male & 51 & 1 & 0 & $\operatorname{sig}$ & 1 & 0 & 1 \\
\hline 6 & Female & 73 & 1 & 2 & muc & 2 & 2 & 2 \\
\hline 7 & Male & 53 & 2 & 2 & tub & 2 & 2 & 2 \\
\hline 8 & Female & 80 & 3 & 2 & poor & 2 & 2 & 2 \\
\hline 9 & Female & 69 & 1 & 0 & tub & 1 & 0 & 0 \\
\hline 10 & Female & 62 & 2 & 3 & tub & 3 & 3 & 3 \\
\hline 11 & Female & 54 & - & - & gastritis & 1 & 0 & 1 \\
\hline
\end{tabular}

pT, depth of tumor invasion; pN, extent of nodal metastasis; according to UICC-TNM classification (20); poor, poorly differentiated adenocarcinoma; tub, tubular adenocarcinoma; sig, signet-ring cell carcinoma; muc, mucinous adenocarcinoma, according to the Japanese Classification of Gastric Cancer (29).

completed in a single test tube and within $1 \mathrm{~h}$. Furthermore, RT-LAMP reaction has been shown to be feasible with crude samples without purification process of RNA (16).

In our previous study, this new technique was introduced to detect minor metastases of gastric cancer by targeting cytokeratin 19 (CK19) gene, which is expressed on cells of epithelial origin, and similar positivity for CK19 mRNA with this technique was obtained compared with conventional RT-PCR from few clinical samples (17). Thus, extensive examination for sensitivity and specificity were designed to illuminate the entity of the positivity. In the current study, clinical specimens were precisely analyzed and the potential of this new technique was evaluated for its sensitivity to detect tumor metastases in LNs during gastric cancer surgeries.

\section{Materials and methods}

Patients. Study participants consisted of 10 patients with primary gastric adenocarcinoma who underwent gastrectomy with curative intent and a patient with corrosive gastritis who underwent total gastrectomy. All participants underwent surgery at the Department of Frontier Surgery, Chiba University Hospital, between June 2006 and June 2009, and agreed with the written protocol of genetic analysis of clinical specimens. Clinicopathological features of all patients enrolled in this study are indicated in Table I. Routine histopathological diagnosis was made using H\&E staining of formalin-fixed, paraffin-sections from the central portion of all dissected LNs, separately from RT-LAMP analysis.

Surgical specimens. Each lymph node was divided in half immediately after resection in operation room. One half was frozen within 30 min after surgery in liquid nitrogen and stored at $-80^{\circ} \mathrm{C}$ until molecular analysis, while the other half was fixed with $10 \%$ buffered formalin and embedded in paraffin for routine $H \& E$ staining. A frozen lymph node was serially sectioned at $5-\mu \mathrm{m}$ intervals. Every set of three serial sections were tested for routine histopathological examination with $\mathrm{H} \& \mathrm{E}$ staining (H\&E), IHC examination with pancytokeratin monoclonal anti-CK19 antibody (AE1/AE3; Dako Cytomation, Denmark), and RT-LAMP amplification targeted CK19 mRNA. Results from H\&E/IHC and RT-LAMP analysis were compared in each set of sections.

RT-LAMP reaction. LAMP reactions were conducted according to the published procedures (13). This reaction relies on auto-cycling strand displacement DNA synthesis that is performed with a DNA polymerase with high-strand displacement activity and 4 specific primers termed inner primers (forward inner primer called FIP and backward inner primer called BIP), and outer primers (called B3 and F3) recognizing 6 independent sequences, and specifically synthesizes a large amount of amplification products, a mixture of stem loop DNAs with several inverted repeats of the target DNA and cauliflower-like structures with multiple loops. Loop primers hybridize to the stem-loops, except for the loops that are hybridized by the inner primer, and prime strand displacement DNA synthesis. When the target DNA is amplified by LAMP reaction, a white precipitate derived from magnesium pyrophosphate (by-product of LAMP reaction) is observed (18). So, the LAMP method does not require special reagents or electrophoresis to detect the amplified DNA. Further, this reaction is accelerated by the use of 2 additional loop primers (called loops F and B) (19). In addition, the LAMP method is also applicable for RNA upon use of reverse transcriptase together with DNA polymerase $(14,15)$. LAMP primers targeting CK19 gene, and glyceraldehydes-3-phosphate dehydrogenase (GAPDH) gene for quantification and proof of the integrity of isolated RNA, were designed using Primer Explorer version 3 (Fujitsu System Solutions, Tokyo, Japan), and those sequences are indicated elswhere (17). RT-LAMP method was carried out with a $25-\mu 1$ total reaction mixture with a Loopamp RNA amplification kit (Eiken Chemical Co., Tokyo, Japan) containing 40 pmol each of inner primers FIP 
and BIP, 5 pmol each of outer primers F3 and B3, 20 pmol each of loop primers loop $\mathrm{F}$ and loop B, 35 pmol dNTPs, $20 \mu \mathrm{M}$ Betamine, $0.5 \mu \mathrm{M}$ Tris- $\mathrm{HCl}(\mathrm{pH} \mathrm{8.8),} 0.25 \mu \mathrm{M} \mathrm{KCl}$, $0.25 \mu \mathrm{M}\left(\mathrm{NH}_{4}\right) \mathrm{SO}_{4}, 0.2 \mu \mathrm{M} \mathrm{MgSO}_{4}, 0.2 \%$ Tween-20, $1.0 \mu \mathrm{l}$ Enzyme Mix [mixture of BstDNA polymerase and avian myeloblastosis virus (AMV) reverse transcriptase] and $5 \mu 1$ RNA at a constant temperature of $63.5^{\circ} \mathrm{C}$ for $60 \mathrm{~min}$. The DNA fragments synthesized by LAMP reaction were detected based on the production of a white precipitate of magnesium pyrophosphate. For real-time turbidity monitoring, absorbance of the reaction mixture at $650 \mathrm{~nm}$ was measured every $6 \mathrm{sec}$. Temperature control for the LAMP reaction and turbidity measurement were performed using a turbidimeter (LA-200 ver. 0.18; Teramecs Co., Kyoto, Japan) especially developed for DNA analysis by LAMP reaction. Either in the case that reaction solution is saturated with magnesium pyrophosphate or solution contains lipids, decline of the turbidity was observed over time (17). Therefore, declines of the turbidity of solution in each measurement interval was estimated as zero increments, and cumulative increments of turbidity at $6 \mathrm{sec}$ intervals were plotted to estimate the gene amplification. The cut-off value for positivity of gene amplification was set at 0.1 , according to the data indicated elsewhere (17).

Preparation of total RNA or lysate from clinical samples. Frozen clinical specimen was ground down with a Multi Beads Shocker (Yasui Kikai, Osaka, Japan). Total RNA was extracted from these samples by RNA assay mini kit (Qiagen, Hilden, Germany) in accordance with the manufacturer's protocol. Part of sections for RT-LAMP analysis from LNs of case 1,9,10,11, indicated in Table I, were preserved for lysate analysis instead of total RNA extraction. For preparation of the lysate, each sample was dissolved in $0.6 \mathrm{ml}$ of lysis buffer [1\% Nonidet-P40 (Nacalai Tesque, Kyoto, Japan)/100 mM Tris- $\mathrm{HCl}(\mathrm{pH}$ 8.0)/0.5 unit/l RNaseOut Ribonuclease Inhibitor (Invitrogen, Carlsbad, CA)]. The solutions were shaken for $1 \mathrm{~min}$ and centrifuged at 15,000 rpm for $10 \mathrm{~min}$. Then, the supernatant was collected and diluted at 5:1 for use.

Estimation of sensitivity for detection of LN metastasis by RT-LAMP analysis. Sensitivity of the LAMP reaction for CK19 cDNA was first determined using CK19 complete cDNA cloned into pOTB7, which was purchased from Invitrogen (Carlsbad). In order to estimate the sensitivity of RT-LAMP analysis for metastatic cancer cells, volumetric presumption of metastatic lesion was performed. Theoretically, a whole LN can be smashed and analyzed for metastasis with the RT-LAMP, thus, sensitivity of this technique to detect metastasis was evaluated with actual volume of metastatic tissues within the sections tested. When all LN metastasis are assumed to be a sphere, actual volume of ITC would be $<4.2 \times 10^{6} \mu \mathrm{m}^{3}$ (maximum diameter $<0.2 \mathrm{~mm}$ ) according to TNM classification of Malignant Tumours by the International Union Against Cancer (20). Thus, volume of metastatic lesion was calculated based on the depth of sections for RT-LAMP and the area of cancer cells detected on H\&E/IHC counterparts. When the calculated volume of lesions were $<4.2 \times 10^{6} \mu \mathrm{m}^{3}$, cancerous volume loaded into the RT-LAMP reaction was considered to be equivalent to that of ITC. Likewise, volume between $4.2 \times 10^{9}$ and $4.2 \times 10^{6} \mu \mathrm{m}^{3}$ (maximum diameter between 0.2 and

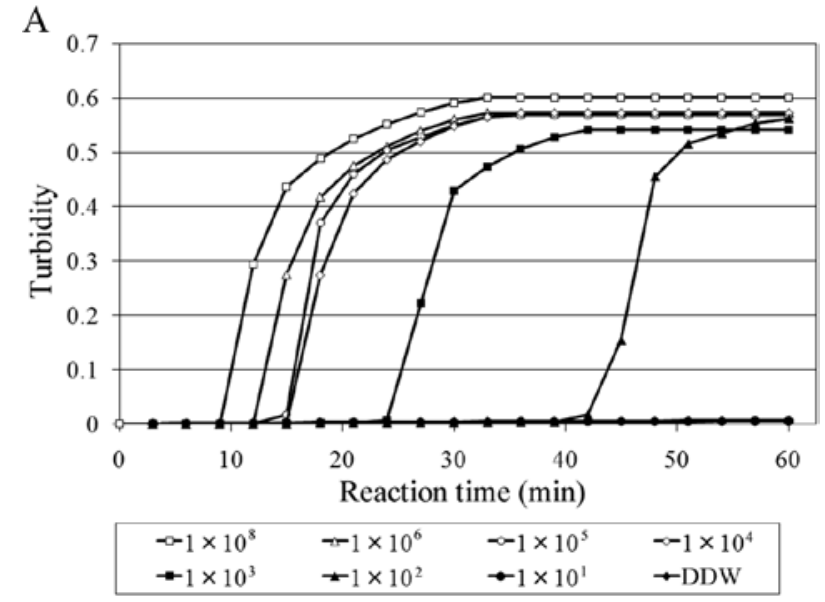

B

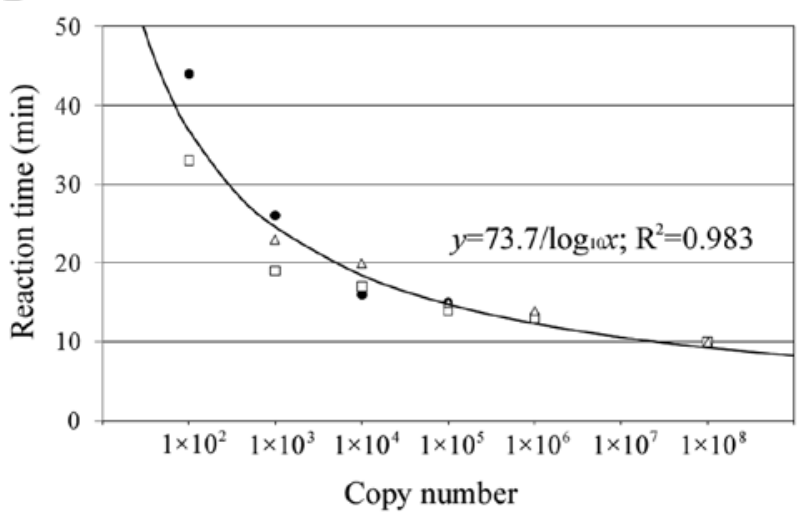

Figure 1. Sensitivity of LAMP technique using a known number of CK19 cDNA copies. (A) Actual turbidity changes of each copy number of CK19 cDNA. (B) Estimated standard curve based on cut-off turbidity of 0.1 .

$2 \mathrm{~mm}$ ) and $>4.2 \times 10^{9} \mu \mathrm{m}^{3}$ (maximum diameter $>2 \mathrm{~mm}$ ) were considered to be micrometastasis (MM) and overt metastasis $(\mathrm{OM})$, respectively.

\section{Results}

Sensitivity of LAMP reaction to detect CK19 cDNA. First, we evaluated the sensitivity of the LAMP reaction using a known quantity of standard CK19-cDNA (Fig. 1A). Standard curves [Reaction time $(\mathrm{min})=73.7 / \log _{10}($ Copy number $) ; \mathrm{R}^{2}=0.983$ ] were produced from serially diluted cloned CK19-cDNA (Fig. 1B), which indicated that the lower limit of detection of CK19-cDNA by the LAMP reaction was $1 \times 10^{2}$ copies. This detection level was achieved within $60 \mathrm{~min}$, whereas $<30 \mathrm{~min}$ was required to detect $1 \times 10^{3}$ copies or more.

Analysis of clinical specimens. Total of $26 \mathrm{LNs}$ were retrieved from surgical specimens of 10 patients with gastric cancer. Histopathological analysis revealed that 21 out of 26 LNs were metastasis-positive and 5 were metastasis-negative (Table I). One mesenteric LN was obtained from a patient undergoing surgery for benign disease for negative control. All of these LNs were serially sectioned and 285 sets of 3 sections were obtained for total RNA analysis and 11 sets were obtained for lysate analysis. The section sets for total RNA analysis were classified into 5 groups according to estimated volume of 


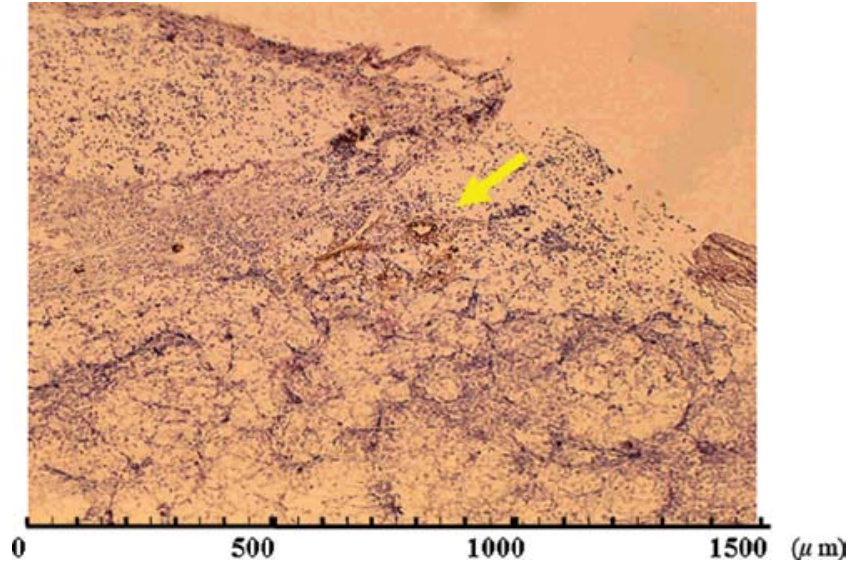

Figure 2. Finding of IHC staining of a section from case 3 indicated in Table I, which was estimated as that containing ITC.

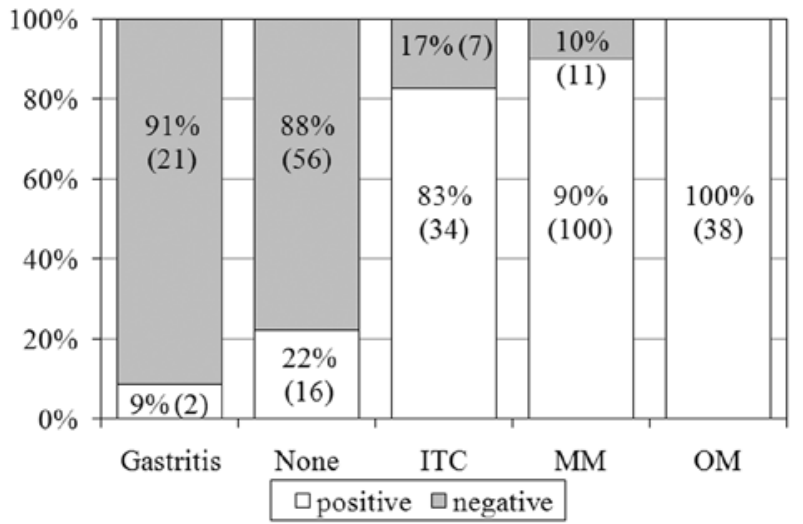

Figure 3. Distribution of RT-LAMP positivity according to volume of metastatic lesions estimated. Actual numbers of section examined are in parentheses. Gastritis, sections of an LN from patients without any malignancies; None, sections of LNs in which no cancer cell were revealed with IHC analysis; ITC, sections of LNs in which cancer cell volume was estimated $<4.2 \times 10^{6} \mu \mathrm{m}^{3} ; \mathrm{MM}$, sections of LNs in which cancer cell volume was estimated between $4.2 \times 10^{6}$ and $4.2 \times 10^{9} \mu \mathrm{m}^{3} ; \mathrm{OM}$, sections of LNs in which cancer cell volume was estimated $>4.2 \times 10^{9} \mu \mathrm{m}^{3}$.

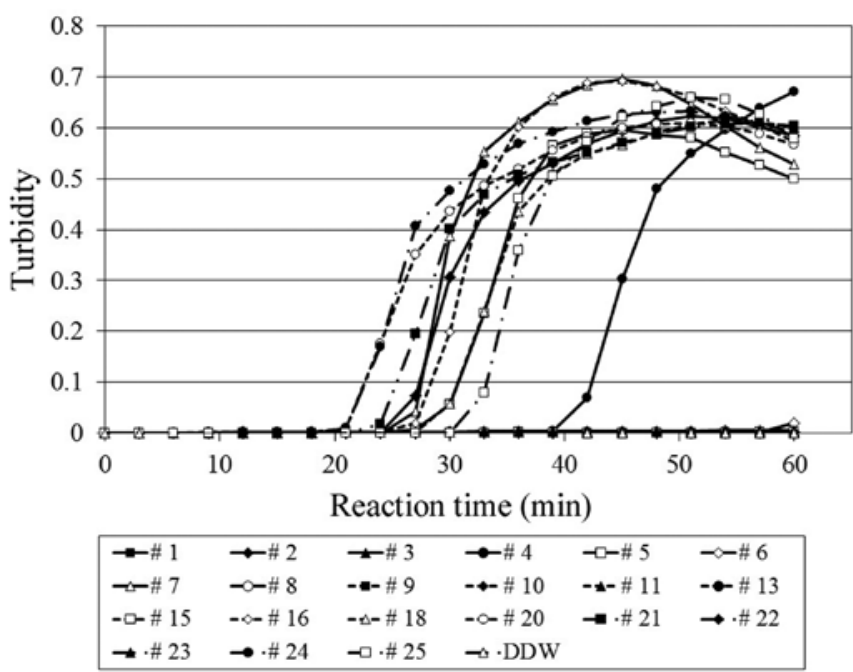

Figure 4. Results of RT-LAMP analysis of all sections of a lymph node from case 3 indicated in Table I. Total of 21 sections were analyzed.
Table II. Distribution of H\&E/IHC and RT-LAMP positivity according to sections within a lymph node from case 3 indicated in Table I.

\begin{tabular}{|c|c|c|c|}
\hline \multirow[b]{2}{*}{ Set no. } & \multirow{2}{*}{$\begin{array}{c}\begin{array}{c}\text { RT-LAMP } \\
\text { analysis }\end{array} \\
\text { Amplification) } \\
\text { time (min) }\end{array}$} & \multicolumn{2}{|c|}{ IHC analysis } \\
\hline & & $\begin{array}{l}\text { No. of metastatic } \\
\text { clusters }\end{array}$ & $\begin{array}{c}\text { Maximum diameter } \\
\text { of clusters }(\mu \mathrm{m})\end{array}$ \\
\hline 1 & - & 1 & 141.5 \\
\hline 2 & 27 & 1 & 139.6 \\
\hline 3 & - & 1 & 97.0 \\
\hline 4 & 42 & $\mathbf{0}$ & - \\
\hline 5 & 31 & 2 & 150.9 \\
\hline 6 & - & 2 & 143.4 \\
\hline 7 & 27 & 3 & 55.2 \\
\hline 8 & - & 0 & - \\
\hline 9 & - & 0 & - \\
\hline 10 & - & 0 & - \\
\hline 11 & - & 0 & - \\
\hline 12 & N/A & 0 & - \\
\hline 13 & - & 0 & - \\
\hline 14 & N/A & 0 & - \\
\hline 15 & - & 0 & - \\
\hline 16 & 29 & $\mathbf{0}$ & - \\
\hline 17 & N/A & $\mathbf{0}$ & - \\
\hline 18 & 30 & 1 & 44.8 \\
\hline 19 & N/A & $\mathbf{0}$ & 35.1 \\
\hline 20 & 25 & 1 & - \\
\hline 21 & 27 & $\mathbf{0}$ & - \\
\hline 22 & - & 0 & - \\
\hline 23 & - & 0 & - \\
\hline 24 & 23 & 0 & - \\
\hline 25 & 33 & 0 & - \\
\hline
\end{tabular}

Estimated ranges of micrometastasis are indicated in bold print. N/A, not applicable due to sample loss.

metastatic lesions, such as sections with $\mathrm{OM}\left(>4.2 \times 10^{9} \mu \mathrm{m}^{3}, 38\right.$ sets), MM (between $4.2 \times 10^{6}$ and $4.2 \times 10^{9}, 111$ ), ITC such as that indicated in Fig. $2\left(<4.2 \times 10^{6}, 41\right)$, sections with no metastatic cells from patients with cancer (None, 72), and sections from patients with gastritis (Gastritis, 23). All of OM sections, 90\% of MM, $83 \%$ of ITC, and $22 \%$ of None showed RT-LAMP positive, whereas $9 \%$ of gastritis were positive for RT-LAMP (Fig. 3). Ten of 11 sets for lysate analysis were from sections with $\mathrm{OM}$ and the rest was that with gastritis.

Mapping of H\&E/IHC and RT-LAMP positivity within an $L N$. Distribution of H\&E/IHC and RT-LAMP positivity of the sections within an LN from case 3 indicated in Table I was determined (Fig. 4) and compared with the positivity of the H\&E/IHC counterparts (Table II). Concordance rate between $\mathrm{H} \& \mathrm{E} / \mathrm{IHC}$ and RT-LAMP was $60 \%(15 / 25)$ on section basis, 


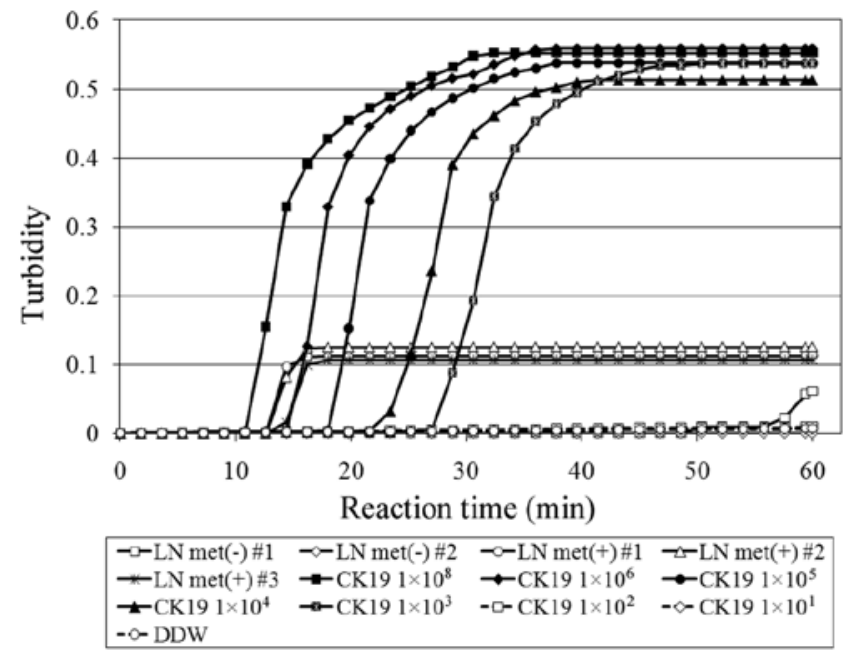

Figure 5. Results of RT-LAMP analysis with lysate from LN sections. LN met(-)\#1, one of the sections with H\&E/IHC negative from case 9 with mucosal cancer; LN met(-)\#2, one of the sections from case 11 without any malignancies; $\mathrm{LN}$ met(+)\#1, one of the sections with $\mathrm{H} \& \mathrm{E} / \mathrm{IHC}$ positive from case 1; LN met(+)\#2, \#3, two of the sections from case 10 with advanced cancer; CK19 1 $10^{1}-10^{8}$, CK19-cDNA solution with indicated copy number for calibration curve; DDW, distilled water as a negative control.

however, clusters of sections showed positivity for H\&E/IHC and/or RT-LAMP (indicated with bold print in Table II) were roughly identical between these two analyses.

RT-LAMP analysis with lysate from sections. Instead of total RNA extraction, lysate were prepared from sections of case $1,9,10,11$, and RT-LAMP analysis were performed with the same procedure (Fig. 5). As a result, samples H\&E/IHC positive from case $1(\mathrm{LN}$ met(+)\#1) and case 10 (LN met(+)\#2 and \#3) with advanced cancer were revealed to be positive within 20 min, whereas a sample with H\&E/IHC negative from case 9 with mucosal cancer (LN met(-)\#1), and a sample from case 11 without any malignancies (LN met(-) \#2) remained negative to the end of the measurement.

\section{Discussion}

SLN concept has been shown to be acceptable to gastric cancer surgeries, and it has a potential to be of great benefit for patients with early stage disease. However, reports of surgeries with much reduced extent of dissection based on this new concept are still rare. Major reasons for this would be the accuracy and rapidity of the detection method of metastasis. False negative rate of SLN biopsies with $\mathrm{H} \& \mathrm{E}$ staining of frozen sections for breast cancer has been reported to be $0-11 \%(2,3,21)$. Detection of $\mathrm{LN}$ recurrences of gastric cancer in early phase after surgery is elaborate, and it will often result in devastating consequences. Thus, lower false negative rate would be anticipated for gastric cancer surgery. Although feasibility studies with IHC or quantitative RT-PCR have shown false negative rate of $0-4 \%$ (22-24), these procedures require considerable time and are hardly acceptable during surgeries.

Recently, Notomi and coworkers reported that a novel DNA amplification technique, LAMP, and rapid diagnostic procedures for various infectious diseases had been developed.
It showed fairly high sensitivity and specificity to detect viral RNA from crude samples without RNA purification process. We applied this technique for diagnosis of nodal metastasis of gastric cancer targeting CK19 mRNA.

Our present study revealed that the limit to detect CK19 mRNA was $1 \times 10^{2}$ copies and $90 \%$ of micrometastasis could be revealed with this new technique. As a consequence, seven out of 10 patients studied were upstaged according to TNM classification (data not shown). These results are comparable with reports on RT-LAMP analysis for nodal metastasis of other malignancies $(25,26)$, showing relatively lower sensitivities compared with quantitative RT-PCR analysis with the same malignancies (10). Meanwhile, relatively higher false positivity was observed in this analysis (9\% for nodes from patient with gastritis). Pseudogenes of CK19 have been reported to show positivity with RT-PCR analysis, however, it could hardly account for the false positive rate in this study, because only $9 \%$ of samples from the same node showed false positivity. Although contamination, some sort of instability of the reaction, or migration of cells of epithelial origin (27) could affect the false positive rate, it requires further analysis.

Baseline declining over time with the analysis of lysate was another issue. Sedimentation of crude material over time or lysis of fatty ingredients with rise of temperature could be associated with this phenomenon. Cumulative algorithm of increments of turbidity adopted in this study was apparently useful to uncover gene amplification from crude samples by RT-LAMP. Other procedures to detect gene amplification such as fluorescence technique (28) could also be a solution.

Total time required to obtain results for existence of metastasis are one of the most important issues in this study. All of the sections which contain comparable volume for OM showed positivity within $30 \mathrm{~min}$ of RT-LAMP reaction. One minute of vortexing and $5 \mathrm{~min}$ of centrifugation of samples need to precede the reaction, thus, a total of $40 \mathrm{~min}$ are required to the judgement. When metastases including MM are evaluated, the reaction time is $60 \mathrm{~min}$, thus, total of $70 \mathrm{~min}$ are required. Although it is rather long, it could be tolerable to most of surgeons, taking duration required for routine histopathological frozen section analysis with $H \& E$ staining in our hospital (40-60 min), into account.

Although couple of issues remain to be further studied, this new technique shows both high and rapid detectability of nodal metastasis of gastric cancer, and easy operability which can be completed within operating room. Therefore, it has a potential to be an alternative to the rapid histopathological diagnosis using frozen section for nodal metastasis in near future.

\section{References}

1. Morton DL, Wen DR, Wong JH, et al: Technical details of intraoperative lymphatic mapping for early stage melanoma. Arch Surg 127: 392-399, 1992.

2. Krag DN, Weaver DL, Alex JC and Fairbank JT: Surgical resection and radiolocalization of the sentinel lymph node in breast cancer using a gamma probe. Surg Oncol 2: 335-340, 1993.

3. Giuliano AE, Kirgan DM, Guenther JM and Morton DL: Lymphatic mapping and sentinel lymphadenectomy for breast cancer. Ann Surg 220: 391-398, 1994.

4. Kitagawa Y, Fujii H, Mukai M, et al: The role of the sentinel lymph node in gastrointestinal cancer. Surg Clin North Am 80: $1799-1809,2000$ 
5. Tsioulias GJ, Wood TF, Morton DL and Bilchik AJ: Lymphatic mapping and focused analysis of sentinel lymph nodes upstage gastrointestinal neoplasms. Arch Surg 135: 926-932, 2000.

6. Hayashi H, Ochiai T, Mori M, et al: Sentinel lymph node mapping for gastric cancer using a dual procedure with dye- and gamma probe-guided techniques. J Am Coll Surg 196: 68-74, 2003.

7. Okada Y, Fujiwara Y, Yamamoto H, et al: Genetic detection of lymph node micrometastases in patients with gastric carcinoma by multiple-marker reverse transcriptase-polymerase chain reaction assay. Cancer 92: 2056-2064, 2001.

8. Matsumoto M, Natsugoe S, Ishigami S, et al: Lymph node micrometastasis and lymphatic mapping determined by reverse transcriptase-polymerase chain reaction in pN0 gastric carcinoma. Surgery 131: 630-635, 2002.

9. Ajisaka $\mathrm{H}$ and Miwa K: Micrometastases in sentinel nodes of gastric cancer. Br J Cancer 89: 676-680, 2003.

10. Kubota K, Nakanishi H, Hiki N, et al: Quantitative detection of micrometastases in the lymph nodes of gastric cancer patients with real-time RT-PCR: a comparative study with immunohistochemistry. Int J Cancer 105: 136-143, 2003.

11. Ishii T, Fujiwara Y, Ohnaka S, et al: Rapid genetic diagnosis with the transcription-reverse transcription concerted reaction system for cancer micrometastasis. Ann Surg Oncol 11: 778-785, 2004

12. Sonoda H, Yamamoto K, Kushima R, Okabe H and Tani T: Detection of lymph node micrometastasis in gastric cancer by MUC2 RT-PCR: usefulness in pT1 cases. J Surg Oncol 88: 63-70, 2004.

13. Notomi T, Okayama H, Masubuchi H, et al: Loop-mediated isothermal amplification of DNA. Nucleic Acids Res 28: E63, 2000.

14. Parida M, Posadas G, Inoue S, Hasebe F and Morita K: Real-time reverse transcription loop-mediated isothermal amplification for rapid detection of West Nile virus. J Clin Microbiol 42: 257-263, 2004.

15. Ushio M, Yui I, Yoshida N, et al: Detection of respiratory syncytial virus genome by subgroups-A, B specific reverse transcription loop-mediated isothermal amplification (RT-LAMP). J Med Virol 77: 121-127, 2005.

16. Visser M, Jiwa M, Horstman A, et al: Intra-operative rapid diagnostic method based on CK19 mRNA expression for the detection of lymph node metastases in breast cancer. Int J Cancer $122: 2562-2567,2008$
17. Horibe D, Ochiai T, Shimada H, et al: Rapid detection of metastasis of gastric cancer using reverse transcription loop-mediated isothermal amplification. Int J Cancer 120: 1063-1069, 2007.

18. Mori Y, Nagamine K, Tomita N and Notomi T: Detection of loop-mediated isothermal amplification reaction by turbidity derived from magnesium pyrophosphate formation. Biochem Biophys Res Commun 289: 150-154, 2001.

19. Nagamine $K$, Hase $T$ and Notomi $T$ : Accelerated reaction by loop-mediated isothermal amplification using loop primers. Mol Cell Probes 16: 223-229, 2002.

20. Sobin LH and Wittekind C: TNM classification of malignant tumours. Wiley-Liss, New York, 2002.

21. Miltenburg DM, Miller C, Karamlou TB and Brunicardi FC: Meta-analysis of sentinel lymph node biopsy in breast cancer. J Surg Res 84: 138-142, 1999.

22. Miwa K, Kinami S, Taniguchi K, Fushida S, Fujimura T and Nonomura A: Mapping sentinel nodes in patients with earlystage gastric carcinoma. Br J Surg 90: 178-182, 2003.

23. Karube T, Ochiai T, Shimada H, Nikaidou T and Hayashi $\mathrm{H}$ : Detection of sentinel lymph nodes in gastric cancers based on immunohistochemical analysis of micrometastases. J Surg Oncol 87: 32-38, 2004.

24. Arigami T, Natsugoe S, Uenosono Y, et al: Evaluation of sentinel node concept in gastric cancer based on lymph node micrometastasis determined by reverse transcription-polymerase chain reaction. Ann Surg 243: 341-347, 2006.

25. Tsujimoto M, Nakabayashi K, Yoshidome K, et al: One-step nucleic acid amplification for intraoperative detection of lymph node metastasis in breast cancer patients. Clin Cancer Res 13: 4807-4816, 2007.

26. Maeda J, Inoue M, Nakabayashi K, et al: Rapid diagnosis of lymph node metastasis in lung cancer with loop-mediated isothermal amplification assay using carcinoembryonic antigenmRNA. Lung Cancer 65: 324-327, 2009.

27. Fraggetta $F$ and Vasquez E: Epithelial inclusion in axillary lymph node associated with a breast carcinoma: report of a case with a review of the literature. Pathol Res Pract 195: 263-266, 1999.

28. Tomita N, Mori Y, Kanda H and Notomi T: Loop-mediated isothermal amplification (LAMP) of gene sequences and simple visual detection of products. Nat Protoc 3: 877-882, 2008.

29. Japanese Gastric Cancer Association: Japanese Classification of Gastric Carcinoma: 2nd English Edition. Gastric Cancer 1: 10-24, 1998. 